\title{
Remoção cirúrgica de lipoma por acesso pré-auricular
}

\author{
Surgical lipoma removal by pre-auricular access \\ Extracción de lipoma quirúrgico por acceso pre-auricular \\ Evelyn de Freitas BOSCAINE ${ }^{1}$ \\ José Carlos Garcia de MENDONÇA ${ }^{2}$ \\ Janayna Gomes PAIVA-OLIVEIRA ${ }^{3}$ \\ Gustavo Silva PELISSARO ${ }^{3}$ \\ Aline Bergman de Souza HERCULANO ${ }^{4}$ \\ Francielly Thomas FIGUEIREDO 5 \\ Daisilene Baena CASTILLO ${ }^{6}$ \\ Ellen Cristina GAETTI-JARDIM ${ }^{7}$
}

${ }^{I}$ Mestre em Clínica Odontológica pela Faculdade de Odontologia da Universidade Federal de Mato Grosso do Sul (Faodo-UFMS)

${ }^{2}$ Chefe do Serviço de Residência em Cirurgia e Traumatologia Bucomaxilofacial do Hospital Universitário Maria Aparecida Pedrossian (HUMAP-EBSERH/UFMS)

${ }^{3}$ Serviço de Residência em Cirurgia e Traumatologia Bucomaxilofacial do Hospital Universitário Maria Aparecida Pedrossian (HUMAP-EBSERH/UFMS)

${ }_{5}^{4}$ Especialista em Cirurgia e Traumatologia Bucomaxilofacial do Hospital Universitário Maria Aparecida Pedrossian (HUMAP-EBSERH/UFMS)

${ }^{5}$ Residente em Cirurgia e Traumatologia Bucomaxilofacial do Hospital Universitário Maria Aparecida Pedrossian (HUMAP-EBSERH/UFMS)

${ }^{6}$ Professora Titular do Serviço de Dor orofacial da Faculdade de Odontologia da Universidade Federal de Mato Grosso do Sul (Faodo-UFMS)

${ }^{7}$ Tutora da Residência em Cirurgia e Traumatologia Bucomaxilofacial do Hospital Universitário Maria Aparecida Pedrossian, Universidade Federal de Mato Grosso do Sul (Faodo-UFMS)

\section{Resumo}

Os lipomas são tumores benignos do tecido adiposo mais frequentes no tronco e nas extremidades do corpo, com incidência baixa na região orofacial. Clinicamente apresentam-se com lesão única ou lobular, assintomático, com crescimento lento, de base séssil, bem delimitados e macios, com coloração amarelada quando mais superficiais, a rósea em regiões mais profundas. $\mathrm{O}$ trabalho tem o objetivo de relatar um caso de lipoma pré-auricular em um paciente de sexo masculino, leucoderma encaminhado ao Serviço de Residência Bucomaxilofacial do Hospital Universitário da Universidade Federal de Mato Grosso do Sul queixando-se de dor na região. O tratamento foi cirúrgico sob anestesia geral, com acesso cirúrgico pré-auricular e exérese total da peça para análise histopatológica. O paciente segue em acompanhamento de 6 meses sem queixas ou alterações funcionais configurando até o momento o sucesso da terapêutica empregada.

Descritores: Lipoma; Neoplasias; Biópsia.

\section{Abstract}

Lipomas are benign tumors of the adipose tissue most frequently found on the trunk and extremities of the body, with low incidence in the orofacial region. Clinically present as a single or lobular lesion, asymptomatic, with slow growth, sessile base, well-defined and soft, yellowish color when more superficial, pinkish in deeper regions. The work aims to report a case of pre-auricular lipoma in a male patient, leucoderma referred to the maxilofacial residency servisse of the University of Mato Grosso do Sul complaining of pain in the region. The treatment was surgical under general anestesia, with pre-auricular surgical access and total excision of the specimen for histopathological analysis. The patient is followed uf for 6 months without complaints or functional changes, configuring the success of the terapy employed so far.

Descriptors: Lipoma; Neoplasms; Biopsy.

\section{Resumen}

Los lipomas son tumores del tejido adiposo que se encuentran com mayor frecuencia en el tronco y las extremidades del cuerpo, com baja incidência en la región orofacial. Clínicamente se presentam con uma lesión única o lobular, asintomática, con crecimiento lento, base séssil, bien delimitada y suave, con un color amarillento cuando es más superficial, el rosado en regiones más profundas. El trabajo tiene como objetivo informar un caso de lipoma preauricular en un paciente masculino, leucoderma remitido al Servicio de Residencia Maxilofacial del Hospital Universitario de la Universidad Federal de Mato Grosso do Sul quejándose de dolor em la región. El tratamento fue quirúrgico bajo anestesia general, con acceso quirúrgico preauricular y escisión total de la muestra para análisis histopatológico. Se sigue al paciente durante 6 meses sin quejas ni cambios funcionales, configurando el éxito de la teparia empleada hasta ahora.

Descriptores: Lipoma; Neoplasias; Biopsia.

\section{INTRODUÇÃO}

O lipoma é um tumor mesenquimal benigno, considerado um dos mais comuns dos tecidos moles. Ocorre, principalmente, no tronco nas porções proximais das extremidades do corpo, na região da cabeça e pescoço, com incidência de 15 a $20 \%$ dos casos. Quando apresentado em região oral e maxilofacial é relativamente raro, tendo uma incidência relativa de $1 \%$ a $4 \%$ dos tumores que acometem esta região ${ }^{1}$. O lipoma não apresenta etiologia esclarecida, porém, acredita-se que alguns fatores como endocrinopatias, alcoolismo e histórico de trauma e infecções na região acometida estejam associados a esse tumor ${ }^{2}$.

Normalmente se apresentam como lesões de crescimento lento, assintomáticas, de base séssil ou pediculada, única ou lobulada, sendo geralmente circundadas por uma cápsula fibrosa. Clinicamente, podem se apresentar como uma lesão única ou lobular. Quando intraorais, localizam-se sob a mucosa em cerca de $50 \%$ dos casos. Embora de etiologia incerta, alguns autores sugerem como fatores causais as alterações endócrinas, a hereditariedade, o trauma e a infecção local ${ }^{3}$.

De acordo com aspectos histopatológicos, os lipomas podem ser classificados em lipomas simples e variantes, como fibrolipoma, condrolipoma, osteolipoma, lipomas intermuscular e intramuscular, angiolipoma, miolipoma, mielolipoma, lipoblastoma benigno, lipoma condroide, lipoma de células fusiformes, lipoma pleomórfico e sialolipoma). O lipoma simples e o fibrolipoma são as variantes histopatológicas mais comuns ${ }^{4}$.

O diagnóstico final somente é obtido por meio de biópsia incisional ou excisional, seguido de análise histológica. Uma característica importante é que a lesão tende a flutuar quando colocado na solução de formol a $10 \%$. Tal evento acontece pelo fato de a gordura ser menos densa que a solução 
fixadora. O tratamento consiste na excisão cirúrgica e casos de recidiva são raramente relatados ${ }^{5}$.

O objetivo deste trabalho é relatar o caso de um lipoma de face na região pré-auricular esquerda tratado com excisão cirúrgica e biópsia por meio de acesso extraoral pré-auricular de Ellis.

\section{CASO CLÍNICO}

Paciente sexo masculino, 35 anos, leucorderma chegou a clínica odontológica da Faculdade de Odontologia da Universidade Federal de Mato Grosso do Sul (FADO/UFMS) no setor de Disfunção Temporomandibular (SERDOF/DTM) com a queixa de dor aos movimentos excursivos mandibulares e a presença de um aumento volumétrico em região pré-auricular do lado esquerdo (Figura 1). Em exame clínico e imaginológico não constatou lesão ou disfunção da Articulação temporomandibular (Figura 2). Paciente foi encaminhado para o setor de Cirurgia e Traumatologia Bucomaxilofacial que conferiu após avaliação dos mesmos exames com provável diagnóstico de lipoma pré-auricular do lado esquerdo. Como plano de tratamento optou-se em realizar excisão cirúrgica da lesão e envio da peça para análise histopatológica por meio do acesso cirúrgico pré-auricular (Figuras 3 e 4). Em exame histopatológico constatou-se lipoma de face em região pré-auricular (Figura 5). Após cirurgia e 6 meses de acompanhamento, o paciente não apresenta assimetria facial, sintomatologia e indícios de recidiva.

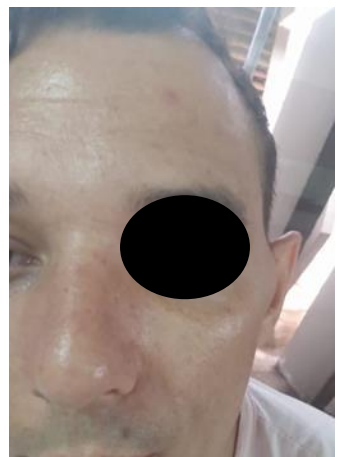

Figura 1: Aspecto clínico inicial. Aumento volumétrico em região préauricular a esquerda.

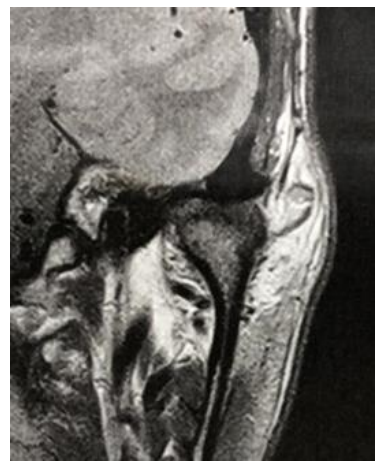

Figura 2: Aspecto Imaginológico. Aumento volumétrico em região préauricular a esquerda.

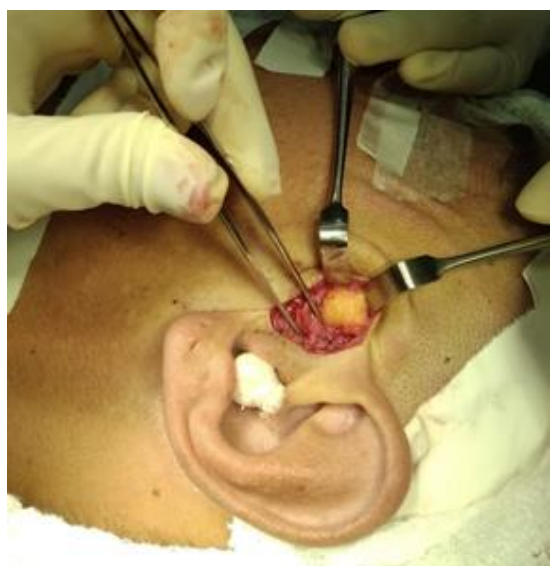

Figura 3: Acesso cirúrgico de Ellis, para remoção da lesão e envio da peça cirúrgica para análise histopatológica.

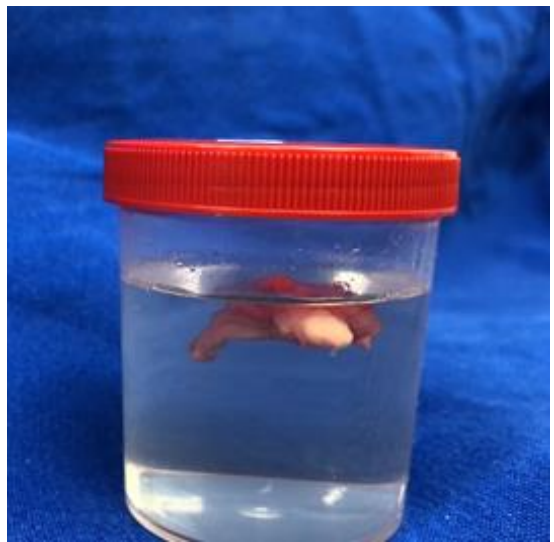

Figura 4: Peça removida e colocada em solução de formol.

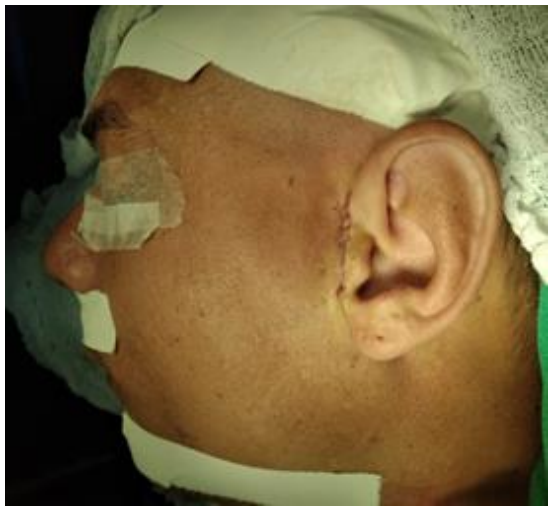

Figura 5: Aspecto clínico pós-operatório imediato. Sutura intradérmica em posição.

\section{DISCUSSÃO}

Os lipomas são as neoplasias mais comuns dos tecidos moles, no entanto são poucos comuns na região de face, principalmente na região pré-auricular conforme o relato de caso. Estudos mais recentes relatam que a região maxilofacial é pouco afeta pelos lipomas e que são mais comuns em homens ${ }^{6,7}$. Em um estudo realizado em 2014 os lipomas estão mais relacionados ao sexo masculino, caucasianos, sem histórico familiar, numa média de idade que varia entre 49 e 60 anos, sem preferência pelo lado direito ou esquerdo, mas parecem estar associados a uma desordem endócrina, trauma ou obesidade, porém relatou-se neste mesmo estudo um caso clínico de uma mulher negra de 32 anos com lipoma em região 
parotídea ${ }^{6}$. Porém, em estudo de 14 casos Mello et al. ${ }^{11}$ observaram maior prevalência em mulheres ${ }^{11}$, em razão da maior quantidade de tecido adiposo quando comparadas aos homens. Não há um consenso na literatura sobre predominância de gênero: alguns autores ${ }^{1,8}$ citam ser mais prevalente em homens, e outros relatam igual distribuição dos lipomas bucais entre os sexos, corroborando assim os nossos estudos atuais. Um caso foi relatado em 2015 de um paciente jovem de 21 anos, sexo masculino, que apresentou um lipoma solitário em região retromandibular esquerda, que procurou atendimento médico após sentir dificuldade de movimento do pescoço $^{7}$. Raramente os lipomas acometem a cavidade bucal, mas quando ocorrem afeta preferencialmente a mucosa, região de vestíbulo, assoalho de boca, gengiva e palato, podendo ainda possuírem localização incomum nos espaços faciais ${ }^{8}$.

Com relação a idade de maior prevalência da lesão, a literatura mostra estar entre a terceira e quinta década de vida, sendo as crianças raramente acometidas, mas quando o são, se dá por volta do sétimo ano de vida ${ }^{9}$. É corriqueiro o surgimento de lipomas de tamanho inferior a $3 \mathrm{~cm}$, porém, por vezes algumas dessas lesões podem chegar a grandes proporções, tendo capacidade ainda de levar a deformidades orofaciais e distúrbios fonéticos em casos de lipoma intraoral. O lipoma apresenta como características clínicas: nódulo móvel, séssil ou pediculado, de consistência mole, bem circunscrito, de coloração amarelada ${ }^{1-4}$, de dimensões variadas, confirmando os mesmos achados clínicos encontrados em nosso relato. O paciente do caso em questão relatou que não notou o início de um crescimento nodular na região pré-auricular, apenas após a evolução da lesão e consequente deformidade facial e prejuízo da estética, corroborando com a literatura que torna claro a controvérsia da etiologia dos lipomas sendo possível que o mesmo esteja relacionado com doenças genéticas, metabólicas e endócrinas, ocorrendo também após casos de trauma na região de ocorrência, hereditariedade, diabetes, radiação ionizante e ainda tratamento com corticoides $^{1-3,6,10,11}$.

O tratamento escolhido na literatura é o tratamento de exérese total da lesão, através da biópsia excisional, corroborando com o nosso caso clínico e que o índice de recidiva é baixo e o prognóstico da lesão é bom.

\section{CONCLUSÃO}

Mesmo na presença de uma lesão em região nobre da face a remoção da mesma se mostrou um sucesso sem complicações ou sequelas e o paciente segue aos cuidados da equipe sem queixas configurando até o momento sucesso da terapêutica.

\section{REFERÊNCIAS}

1. Costa DFN, Inaoka SD, Silverira KG, Souza NL, Santos LAM. Tratamento cirúrgico de lipoma: relato de dois casos. Rev cir traumatol bucomaxilo-fac. 2017;17(3):25-8.

2. Resende R, Meirelles M, Varella R. Remoção cirúrgica de lipoma de grande proporção: relato de caso. Rev cir traumatol buco-maxilo-fac. 2013;13(2):37-42.

3. Osterne RLV, Lima-Verde RMB, Turatti E, Cavalcante R. Lipoma de cavidade oral: um estudo de 101 casos em uma população brasileira. J Bras Patol Med Lab. 2019;55(2):148-59.

4. Tenório JR, Paiva KM, Nogueria PTB, Oliveira e Silva ED. Exérese de Extenso Lipoma em Região Submandibular: Relato de caso. Rev cir traumatol buco-maxilo-fac. 2013;13(3):37-40.

5. Menezes RO, Tavares SS, Peixoto TS, Aragão MS, Godoy GP. Unusual facial lipoma. RGO. 2014;62(4):425-30.

6. Mandarino SCA, Guimarães, MA, Coutinho MA. Lipoma em região parotídea: Relato de caso. Rev cir traumatol buco-maxilo-fac. 2014;14(3):33-8.

7. Shetty N, Shabari UB, Jaydeep NA, Patnaik P. Solitary lipoma in the retromandibular region. Indian J Dent. 2015;6(1):49-52.

8. Chuengue EK, Oliveira WD, Silva JPQ, Freitas DL. Remoção cirúrgica de lipoma volumoso em região temporal: Relato de caso. Rev Saberes. 2019;10(1).

9. Chia CY, Rovaris DA, Fontana R. Lipoma gigante do coxim adiposo bucal: relato de caso e revisão da literatura. Rev Bras Cir Plast. 2016;31(1):112-17.

10. de Souza Batista FR, Figueira JA, Lustosa RM, Eidt JM, Piacentini M, Pavan ÂJ. Lipoma in the Face Associated With Maxillofacial Trauma. J Craniofac Surg. 2017;28(1):295-96.

11. Mello DF, Manica MZ, Helene AJ. Lipomas gigantes: Séries de 14 casos. Rev Bras Cir Plast. 2015;30(1):33-7.

\section{CONFLITO DE INTERESSES}

Os autores declaram não haver conflitos de interesse.

\section{AUTOR PARA CORRESPONDÊNCIA}

\author{
Evelyn de Freitas Boscaine \\ Faculdade de Odontologia - Faodo \\ Fundação Universidade de Mato Grosso do Sul \\ Avenida Costa e Silva, s/n - Bairro Universitário \\ 79070-900 Campo Grande - MS, Brasil \\ e-mail: evelynboss@ hotmail.com
}

